\title{
Balancing Energy Consumption in Wireless Sensor Networks Using Biogeography Based Optimization Algorithm and Fuzzy Approach
}

\author{
Basim Abood ${ }^{1}$, Aliaa Hussien ${ }^{2}$ and $\mathrm{Yu} \mathrm{Li}^{1^{*}}$,Desheng Wang ${ }^{1}$ \\ ${ }^{1}$ National Laboratory for Optoelectronics, Huazhong University of Science and Technology Wuhan, \\ China 430074 \\ bas_eng84@ymail.com; * hustlyu@hust.edu.cn;dswang@hust.edu.cn; \\ ${ }^{2}$ Baghdad University, College of Science, Department of astronomy. \\ aliaa1987@yahoo.com;
}

\begin{abstract}
Extending the lifetime of the energy constrained wireless sensor networks is a crucial challenge in wireless sensor networks (WSNs) research. When designing a WSN infrastructure Resource limitations have to be taken into account. The inherent problem in WSNs is unbalanced energy consumption, characterized by multi hop routing and a many-to-one traffic pattern. This uneven energy dissipation can significantly reduce network lifetime. This paper proposes a new routing method for WSNs to extend network lifetime using a combination of a fuzzy approach and Biogeography Based Optimization (BBO) algorithm. Determine an optimal routing path from the source to the destination by favoring the highest remaining battery power, and the lowest distance (minimum number of hops) to the sink. To demonstrate the effectiveness of the proposed method in terms of balancing energy consumption and maximization of network lifetime, we compare our approach with the BBO search algorithm and fuzzy approach using the same routing criteria. Simulation results demonstrate that the network lifetime is significantly increased by employing the proposed routing method.
\end{abstract}

\section{Indexing terms/Keywords}

Biogeography Based Optimization (BBO) algorithm, fuzzy logic, network lifetime, routing, wireless sensor networks (WSNs).

\section{Academic Discipline And Sub-Disciplines}

Wireless Sensor Networks, Artificial Intelligence;

\section{SUBJECT CLASSIFICATION}

Routing, Artificial Intelligence

\section{TYPE (METHOD/APPROACH)}

New Routing Approach For Wireless Sensor Networks

\section{Council for Innovative Research}

Peer Review Research Publishing System

Journal: INTERNATIONAL JOURNAL OF COMPUTERS \& TECHNOLOGY

Vol. 15 , No. 3

www.ijctonline.com, editorijctonline@gmail.com 


\section{INTRODUCTION}

Sensor nodes in the large-scale data-gathering networks are generally powered by small and inexpensive batteries in expectation of surviving for a long period [1]. Each sensor node makes its decisions based on its mission, the information it currently has, knowledge of its computing, communication, and energy resources. Due to limitations in the communication range, sensor nodes transmit their sensed data through multiple hops. Each sensor node acts as a routing element for other nodes for transmitting data. Energy is therefore of utmost importance in power- constrained datagathering sensor networks. Energy consumption should be well managed to maximize the network lifetime [2]. Unbalanced energy consumption is an inherent problem in WSNs characterized by the multi-hop routing and many-to one traffic pattern. This uneven energy dissipation can significantly reduce network lifetime. Generally in routing algorithm, the best path is chosen for transmission of data from source to destination. Over a period of time, if the same path is chosen for all communications in order to achieve battery performance in terms of quick transmission time, then those nodes on this path will get drained fast [1, 3, 4]. In most applications of WSNs, sensor nodes are densely deployed in large areas. Once deployed, nodes can never be recharged or replaced. After depleting their energy, nodes turn to die and stop working. Since networks cannot accomplish assigned missions after nodes die. The lifetime of WSNs is a crucial parameter when evaluating the performance of routing protocols [4]. Fig. 1 shows the network partition (one part of the network may become disconnected from the destination) due to the death of some sensor nodes.

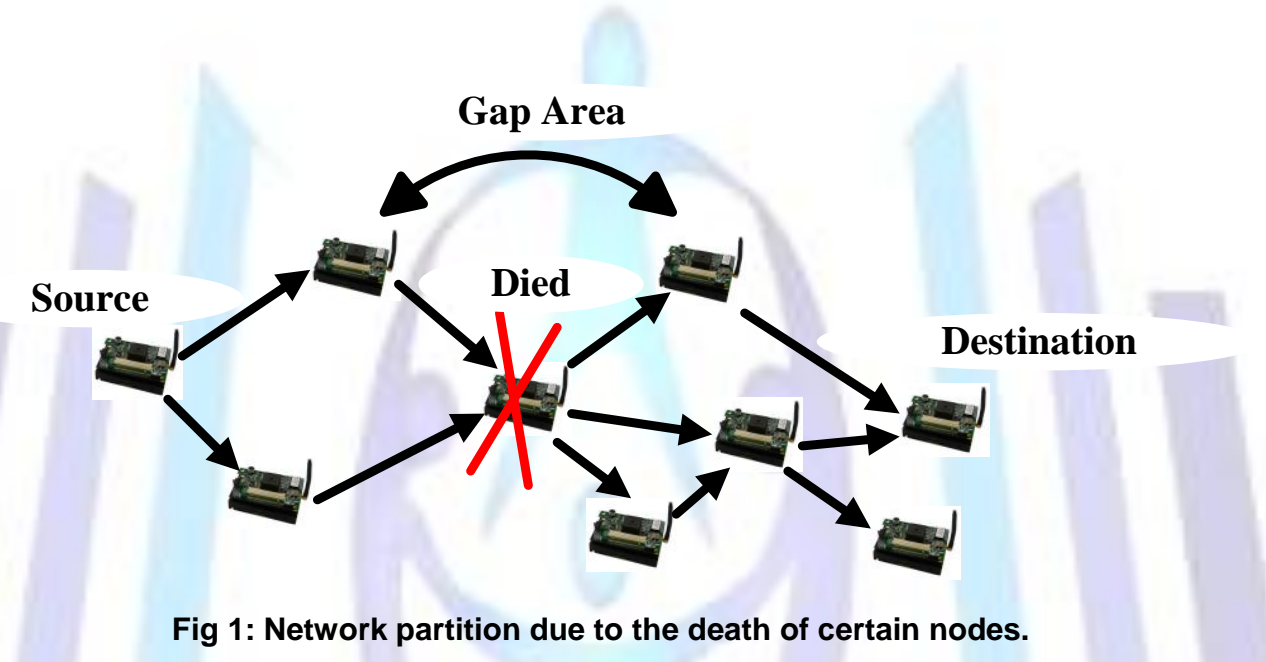

In traditional optimal path routing schemes over WSNs, each node selects specific nodes to relay data according to some criteria in order to maximizing network lifetime. Due to this conception, the lifetime problem in WSNs has received significant attention in the recent past.

Chang et al. in [2] proposed a shortest cost path routing algorithm for maximizing network lifetime based on link costs that reflect both the communication energy consumption rates and the residual energy levels.

The work in [5] addressed the issue of lifetime analysis and estimation for wireless sensor networks in which the sensor nodes are deployed at desired locations. Instead of trying out various probability basis, to apply an interval type-2 fuzzy logic system (FLS) for lifetime analysis and estimation in a wireless sensor network is proposed.

The authors [6] proposed a hybrid routing method for a heterogeneous WSN to prolong the lifetime of the network. The hybrid routing combines clustering and multi-hop communication methods, which are nodes placed near to sink perform a multi-hop network and nodes far away from sink perform clusters. In [7], the authors used an ant colony optimization approach to maximize the lifetime of heterogeneous WSNs. In [8] the authors presentedOptimal Forwarding by Fuzzy Inference Systems (OFFIS) for flat sensor networks. The OFFIS protocol selected the best node from candidate nodes in the forwarding paths by favoring the minimum number of hops, shortest path and maximum remaining battery power, etc.

The authors in [9] presented a novel algorithm for routing analysis in WSNs utilizing a fuzzy logic at each node to determine its capability to transfer data based on its relative energy levels, distance and traffic load to maximize the lifetime of the sensor networks. Rana et al. in [10] used A-star algorithm to search optimal route from the source to destination in such a way that, there is a pre-defined minimum energy level for sensor nodes so that sensor node doesn't participate in routing if its residual energy level is below that level.

In this work, a new approach (Biogeography-Based Optimization (BBO) algorithm) for dynamic deployment problem for WSNs is proposed. We considered WSNs which combine mobile and static sensors. It is known that the BBO algorithm and its variants work well for many optimization problems [11]. The BBO algorithm was initially used on dynamic deployment for WSN. Considering the good performance of the algorithm, use of the BBO algorithm seemed an appropriate approach to enable the sensors in the network to obtain a good coverage in two dimensional space with static and mobile nodes.

From the aforementioned literatures, we note that a number of different metrics have been used to prolong the lifetime of the sensor networks. These metrics are as follows: 
- Remaining Energy (RE): A routing protocol that uses this metric would then favour routes that have the largest total energy capacity from source to destination. In other words, nodes having greaterremaining energy participate more than the nodes with limited power [4, 12, and 13]. Fig. 2 shows an example of a small sensor network, where a source node wishes to transmit a packet to a destination node. The numbers inside the nodes indicate the remaining energy capacity of corresponding nodes. In this example, a routing protocol could select path A-D-F since it has the largest total capacity (i.e. 12).

- $\quad$ Minimum Hop $(\mathbf{M H})$ : The basic idea behind this metric is that using the shortest path will result in low end-to-end delays and low resource consumptions, since the smallest number of forwarding nodes will be involved [9, 12, and 13]. In Fig. 2 a routing protocol, under this criterion, could select the path B-E which has the minimum hop (i.e. 3).

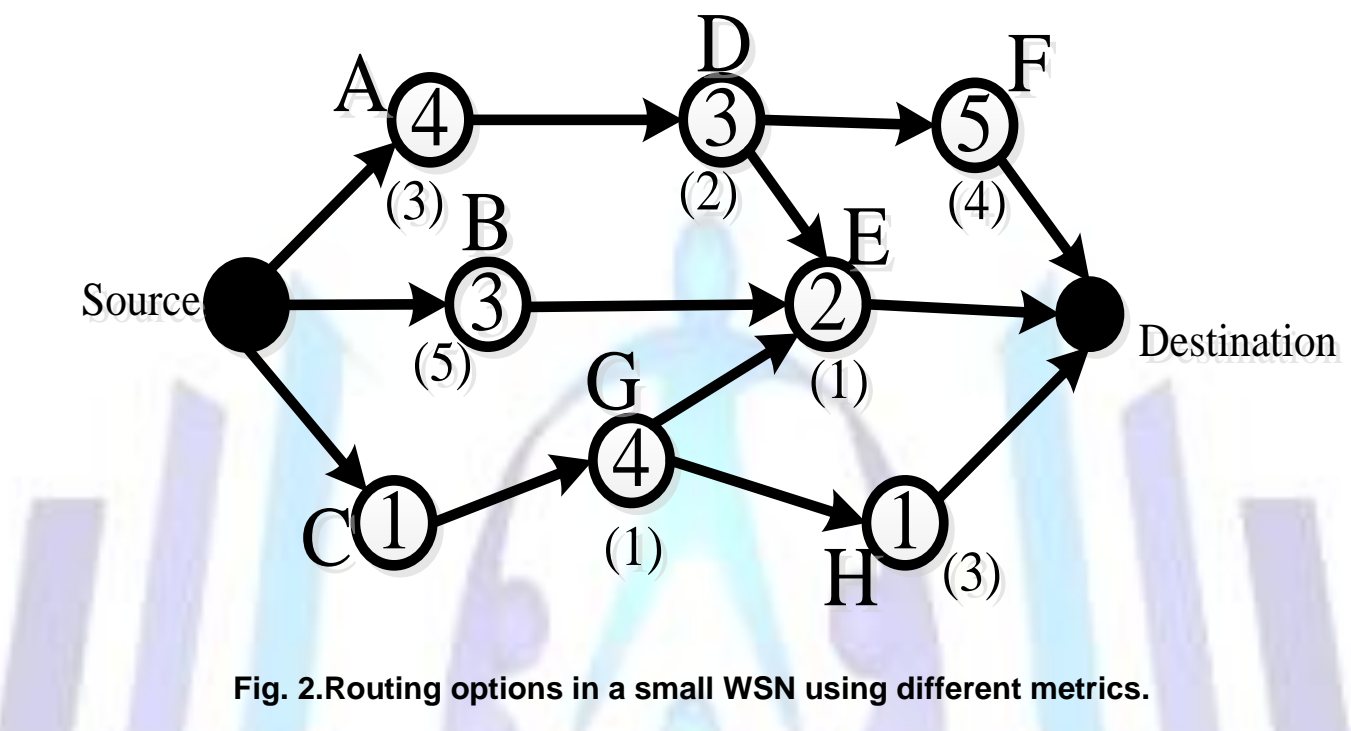

In this paper, the proposed method seeks to investigate the problems of balancing energy consumption and maximizing network lifetime for WSNs. We propose a new approach by combining Fuzzy approach and Biogeography Based Optimization algorithm by selected the select the optimal routing path from source to destination by considering the above criteria (Remaining Energy and Minimum Hop) and balancing between them to lengthen the lifetime of the sensor network as much as possible.

The rest of this paper is organized as follows. In Section 2, the paper describes a brief background of Fuzzy approach and Biogeography Based Optimization algorithm. The routing model for the proposed routing method is presented in Section 3. Performance evaluation is presented in Section 4. Finally, conclusion and discussion are presented in Section 5.

\section{FUZZY APPROACHAND BIOGEOGRAPHY BASED OPTIMIZATION ALGORITHM.}

\subsection{Fuzzy Approach}

The concept of fuzzy logic was introduced by Zadeh in the mid-1960s [14] as an extension of the concept of an ordinary fuzzy set. Since then, its applications have rapidly expanded in adaptive control systems and system identification. It has the advantages of easy implementation, robustness, and ability to approximate to any nonlinear mapping [15]. In fuzzy systems, the dynamic behavior of a system is characterized by a set of linguistic fuzzy rules based on the knowledge of a human expert. These rules are the heart of a fuzzy system and may be provided by experts or can be extracted from numerical data. In either case, the rules that we are interested in can be expressed as a collection of IF-THEN statements (IF antecedents THEN consequents). Antecedents and consequents of a fuzzy rule form the fuzzyinput space and fuzzy output space respectively are defined by combinations of fuzzy sets. Considering a fuzzy system with $p$ inputs and one output with $\mathrm{M}$ rules, then the Lth rule has the form [16]:

$R^{L}:$ IF $x_{1}$ is $F_{1}^{L}$ and $\ldots x_{P}$ is $F_{P}^{L}$ THEN $y$ is $G^{L}$ where $F_{1}^{L} \ldots F_{P}^{L}$ and $G^{L}$

Where $F_{1}^{L} \ldots F_{P}^{L}$ and $G^{L}$ denote the linguistic variables defined by fuzzy sets and $L=1 \ldots M$.

Fig. 3, and fig.4, shows the typical structure of a fuzzy system. It consists of four components namely; fuzzification, rule base, inference engine and defuzzification [14]. 


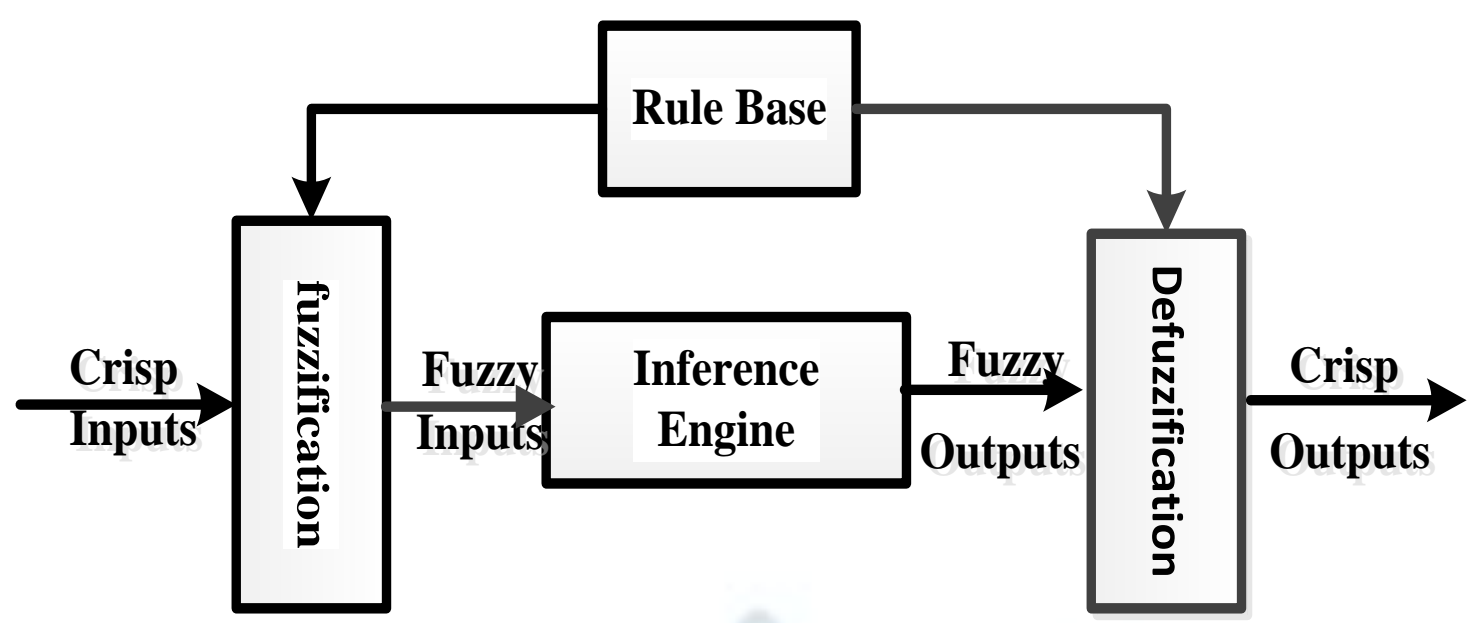

Fig. 3.Typical structure of the fuzzy approach.

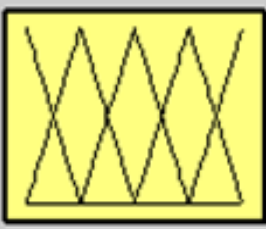

Remaining Energy $\mathrm{RE}(\mathrm{s})(5)$
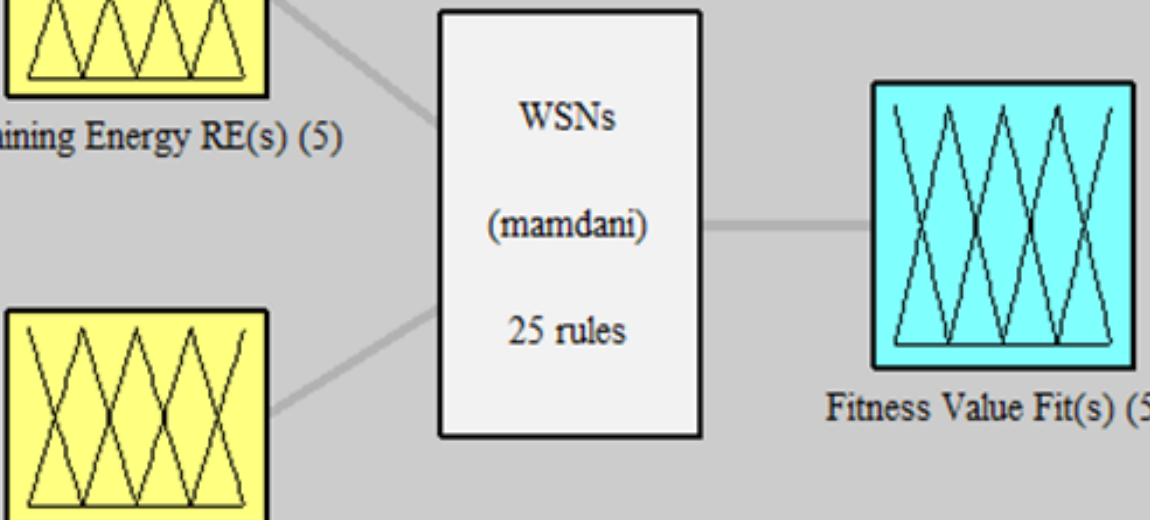

Fitness Value Fit(s) (5)

Normalize Distance D(s) (5)

\section{Fig. 4 fuzzy system Architecture (2 inputs, 1 output, 25 rules)}

The processes of making crisp inputs are mapped to their fuzzy representation in the process called fuzzification.This involves application of membership functions such as triangular, trapezoidal, Gaussian etc. The inference engine process maps fuzzified inputs to the rule base to produce a fuzzy output. A consequent of the rule and its membership to the output sets are determined here. The defuzzification process converts the output of a fuzzy rule into crisp outputs by one of defuzzification strategies [17].

\subsection{Biogeography-Based Optimization algorithm}

The Biogeography-Based Optimization (BBO) algorithm developed by Simon in 2008[18]. The basic premise of this theory is that the rate of change in the number of species on an island critically depends on the balance between the immigration of new species onto the island and the emigration of established species. The BBO algorithm operates in a population of individuals called habitats (or islands). Each habitat represents a possible solution to the problem in hand. The fitness of each habitat is determined by its habitat suitability index (HSI), which is a metric that determines the goodness of a candidate node solution, and each habitat feature is called a suitability index variable (SIV). Good solutions may have a larger number of species, which represents a habitat with a low HSI, than do poor solutions. The immigration rate $\lambda$ and the emigration rate $\mu$ of each habitat are used to probabilistically share information between habitats. These parameters are affected by the number of species $S$ in a habitat. Maximum immigration rate $I$ occur when the habitat is empty and 
decreases as more species are added and maximum emigration rate $E$ occurs when all possible species Smax are present in the habitat. The immigration and emigration rates when there are $S$ species in the habitat are shown in fig 5:

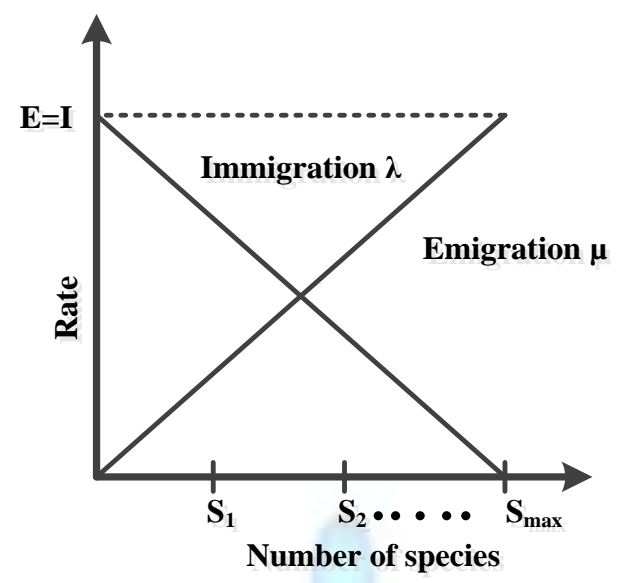

Fig5. Illustration of candidate nodes solutions to some problem

In fig4. The candidate nodes solutions represent as $S_{1}$ represent a low $\mathrm{HSI}$ solution, while $S_{2}$ represent a high $\mathrm{HSI}$ solution. So, in same figure $S_{1}$ represent a habitat with only a few species, while $S_{2}$ represents a habitat with many species. The immigration rate $\lambda_{1}$ for $S_{1}$ will, therefore, be higher than the immigration rate $\lambda_{2}$ for $S_{2}$. The emigration rate $\mu_{1}$ for $S_{1}$ will be lower than the emigration rate $\mu_{2}$ for $S_{2}$. In BBO, $\lambda_{1}$ is the probability that a given independent variable in the $i$-th candidate solution will be replaced; that is, $\lambda_{i}$ is the immigration probability of $x_{i}$. If an independent variable is to be replaced, then the emigrating candidate solution is chosen with a probability that is proportional to the emigration probability $\mu_{i}$. This probability value is usually performed using roulette wheel selection for emigration [18].

$$
P\left(x_{i}\right)=\mu_{i} / \sum_{n=1}^{N} \mu_{n}
$$

For $i=1, \ldots N$, where $N$ is the number of candidate solution in the population.

\section{PROPOSED ROUTING METHOD}

In this paper, the topology of a WSN is modeled as a directed graph G (N, A), where $N$ is the set of nodes, and $A$ is the set of direct links between the nodes. A sink node is responsible for collecting data from all other nodes within its transmission range [19]. The routing schedule is computed by the base station. It calculates optimal routing schedule and broadcasts it. Every node follows this schedule. The process of finding the optimal path, and broadcasting it in the network and sending data from all nodes to the base station by following this routing schedule is repeated in every round. Computation of routing schedule is done dynamically with the consideration of current level of some criteria of each node. For this, normally it may require the nodes to report their criteria periodically to the base station. The base station can then determine the routing schedule based on this updated information. The proposed method assumes that:

- All sensor nodes are randomly distributed in the area and every sensor node is assumed to know its ownposition as well as that of its neighbors and the sink.

- All sensor nodes have the same maximum transmission range and the same amount of initial energy.

One of the important measures of WSN is the network lifetime. For the proposed model, whenever any sensor node runs out of energy, communication links between various sensor nodes and the base station will break. This is considered as the end of the network lifetime. Since the lifetime of each sensor node depends on energy consumption, it is important to preserve residual energy of these nodes in such a way that overall network lifetime is extended. The primary goal of this paper is to design a protocol that will prolong the lifetime of the WSNs through limiting energy cost as well as equal distribution of energy consumption. To achieve this, we make use of both the Fuzzy approach and BBO algorithm. This method uses the process consists of two parts.

\subsection{Implementation of Fuzzy Approach}

In this Section, we focus on describing the major goal of fuzzy approach in proposed protocol. Fuzzy approach is used to calculate the value of the fitness function fit(s) of node $s$ that depends on the remaining energy $R E(s)$ and the distance $D(s)$ to the Sink. Fig.6 shows the proposed method uses five membership functions for each input $R E(s)$, normalize $D(s)$

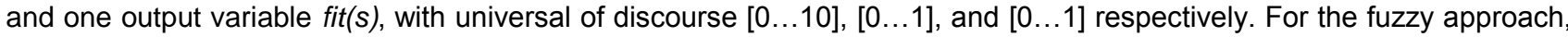
the fuzzified values are processed by the inference engine, which consists of a rule base and various methods to inference the rules. Table I shows the IF-THEN rules used in the proposed method, with a total number of $5^{2}=25 \mathrm{for}$ the 
fuzzy rule base. As an example, IF RE(s) is Very High and $D(s)$ is Very Near THEN Fit(s) is Very Good. All these rules are processed in a parallel manner by a fuzzy inference engine
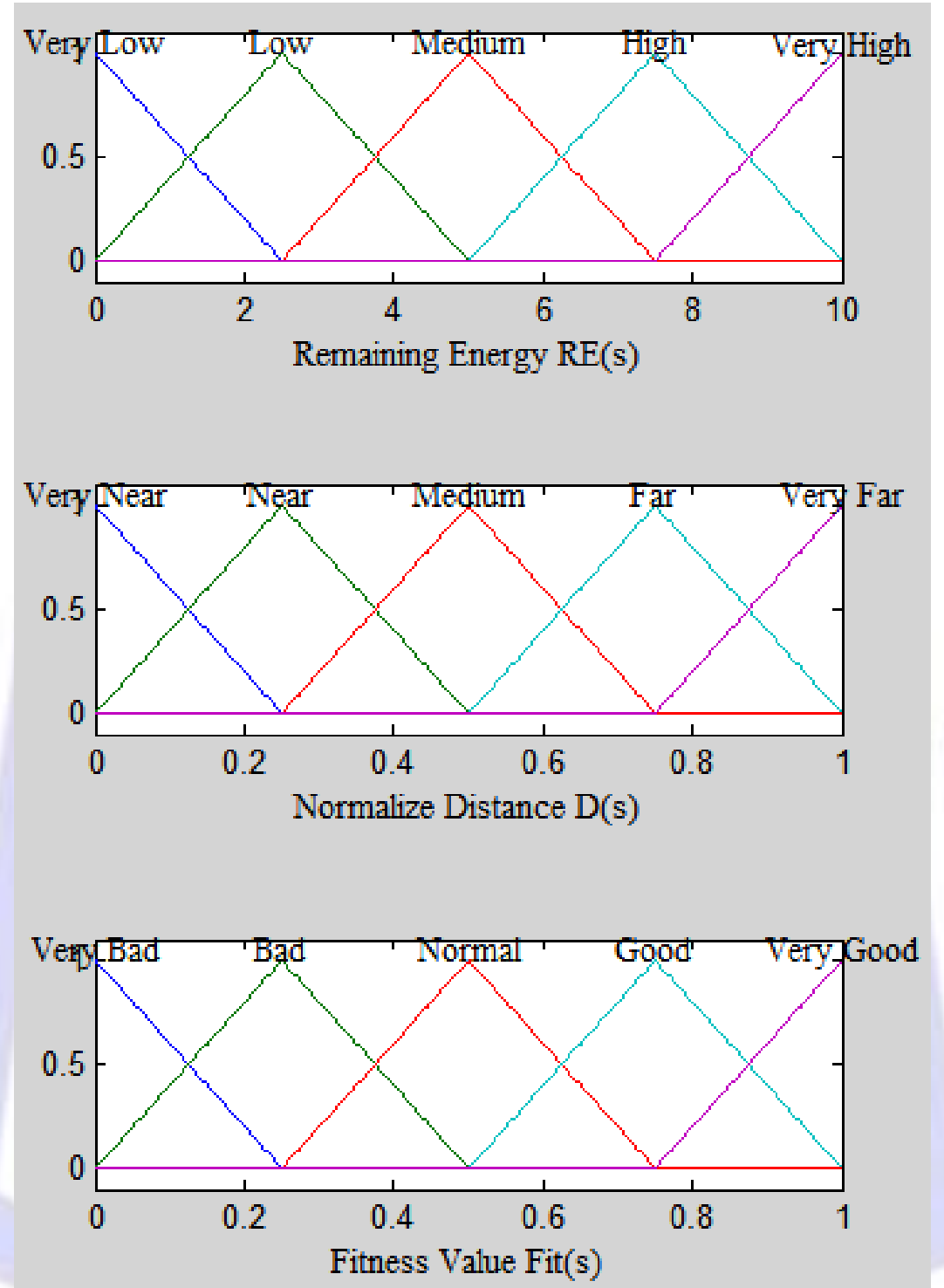

Fig. 6.Membership graph for the inputs (remaining energy and distance to sink) and the output (Fitness value).

Table I IF-THEN Rules

\begin{tabular}{|c|c|c|c|c|c|}
\hline $\begin{array}{l}\text { RE(s) } \\
D(s)\end{array}$ & V. Low & Low & Medium & High & V. High \\
\hline V. Near & Normal & Good & Good & V. Good & V. Good \\
\hline Near & Bad & Normal & Good & V. Good & V. Good \\
\hline Medium & V. Bad & Bad & Normal & Good & Good \\
\hline Far & V. Bad & V. Bad & Bad & Normal & Normal \\
\hline V. Far & V. Bad & V. Bad & Bad & Bad & Bad \\
\hline
\end{tabular}


The defuzzification finds a single crisp output value from the solution fuzzy space. This value represents the fitness function value of node cost (s). Practice defuzzification is done using centre-of-gravity method [20] given by:

$$
f i t(s)=\left(\sum_{k=1}^{n} U_{k} * c_{k}\right) / \sum_{k=1}^{n} U_{k}
$$

Where $U_{k}$ is the output of rule base $k$, and $c_{k}$ is the center of the output membership function.

\subsection{Implementation of BBO Algorithm}

Unbalanced energy consumption is an inherent problem in WSNs characterized by the multi-hop routing and many-toone traffic pattern. This uneven energy dissipation can significantly reduce network lifetime. Generally, in routing algorithm, the best path is chosen for transmission of data from source to destination. Over a period, if the same path is chosen for all communications to achieve battery performance in terms of quick transmission time, then those nodes on this path will drain fast. In this paper, we therefore exploit the BBO algorithm to find the optimal routing path for WSNs. This algorithm seeks to investigate the problems of balancing energy consumption and maximization of network lifetime. BBO seeks to find the optimal routing path from the source basic node to the sink. It selects the best node from candidate nodes (neighbors) in the forwarding paths in favoring the highest remaining energy for each node and the short distance (minimum number of hops) to sink. BBO algorithm considers a tree structure in terms of $(S, F)$, where $S$ is the set of candidate solution nodes in the forwarding path and $F$ is the fitness function, which assigns a fitness value $f(s)$ to each candidate solution node $s \in S$.

The fitness value $f(s)$ is determined depending on the remaining energy of node $s$ and the distance from node $s$ to the sink. The tree node is explored on the basis of its fitness value. Where the candidate solution node that has the highest remaining energy and the lowest distance to the sink will be selected as the best node in the forwarding path to the sink. The fitness function $f(s)$ we used is given as follows:

$$
f(s)=\alpha * R E(s)+1 /(\beta \times D(s))
$$

Where $\mathrm{RE}(\mathrm{s})$ is the remaining energy for node $s, D(s)$ is the distance from node $s$ (minimum number of hops) to the destination, and $\alpha, \beta$ are constant values $(\alpha=0.1$ and $\beta=0.16)$. The candidate nodes in BBO algorithm are selected as the next hops (neighbors) of the source where each employed is nominated to one of these nodes because each employed represents to a candidate node in the following path. All employed compute the fitness functions of their nodes and share this information. According to the equation (2), the evaluates the information taken from all employed and chooses a best node with highest probability $P$ related to its probability value given by the following [18]:

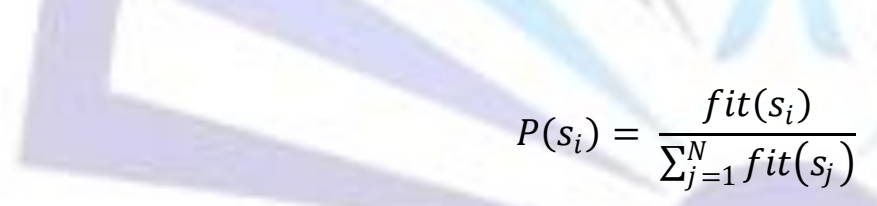

Where $P\left(s_{i}\right)$ is the probability value for node $s_{i}$, fit $\left(s_{i}\right)$ is the fitness value of the node $s_{i}$, and $N$ is the number of candidate solution nodes. The flow chart of the proposed method BBO - Fuzzy is shown in Fig.7.

\section{PERFORMANCE EVALUATION}

To demonstrate the effectiveness of the proposed method in terms of balancing energy consumption and maximizing network lifetime, the method is compared with BBO algorithm and with Fuzzy approach. All these three methods use the same routing criteria namely, the remaining energy, and the minimum hop in selecting the optimal path from the source node to the sink node. Fig. 7 shows the flow chart of the proposed method that is a combination of Fuzzy approach and BBO algorithm to find optimal routing path from start node to the sink node. 


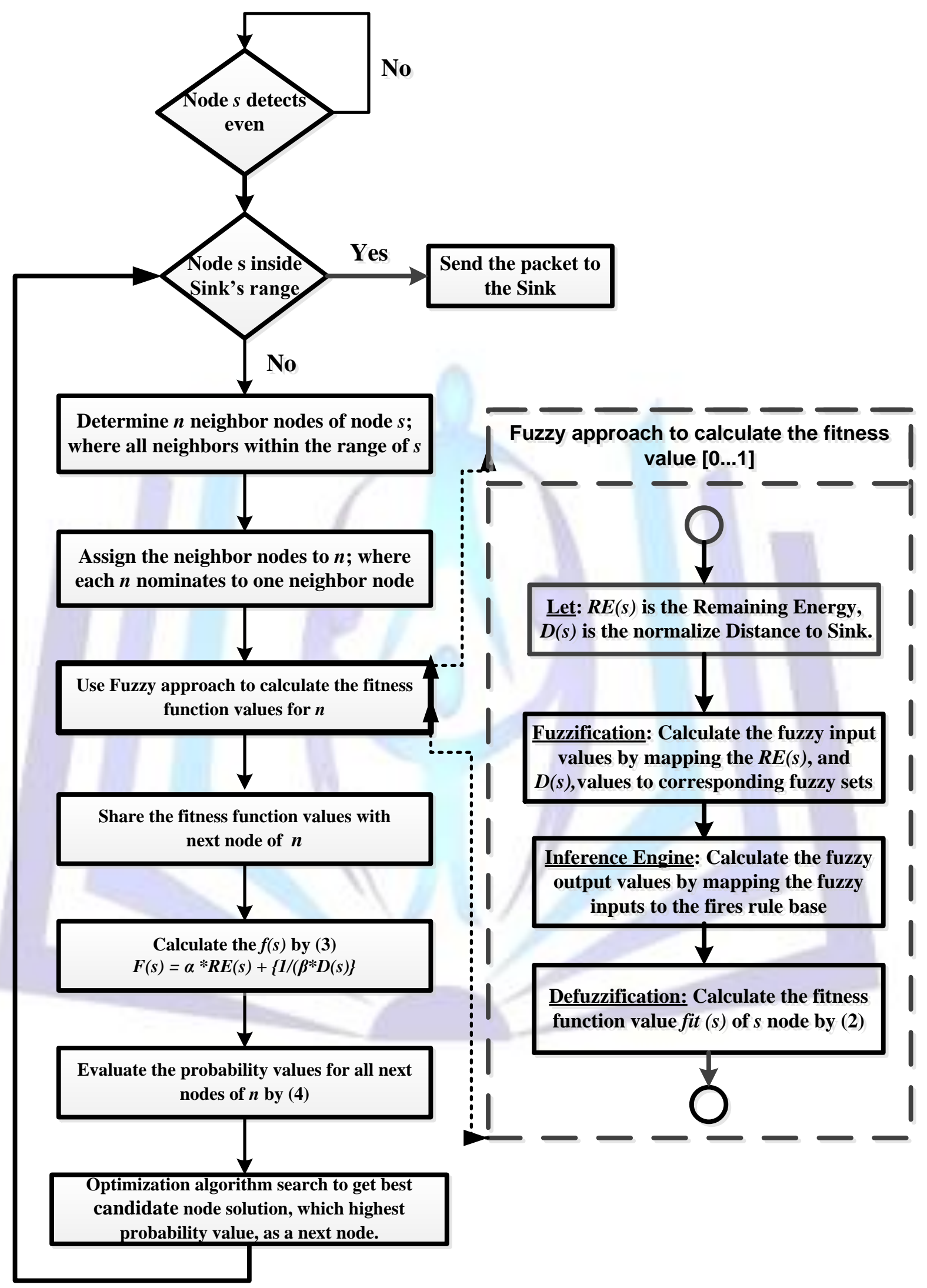

Fig. 7.Flow chart of the proposed algorithm. 


\subsection{Simulation Setup}

The simulations are carried out in MATLAB. 100 sensor nodes are randomly deployed in a $100 \mathrm{~m} \times 100 \mathrm{~m}$ field with sensed transmission limit of $30 \mathrm{~m}$. A data sink node is located at $(90 \mathrm{~m}, 90 \mathrm{~m})$. All sensor nodes have the same initial energy of $0.5 \mathrm{~J}$. The proposed method uses the first order radio model that is largely used in the area of routing protocol evaluation in WSN [21]. According to this model, transmission and receiving costs are characterized by the expressions.The transmission and receiving costs are characterized by the expressions respectively.

$$
E_{n} T(k)=E_{\text {elec }} * k+E_{\text {amp }} * k \cdot d^{2} \quad \text { and } \quad E_{n} R(k)=E_{\text {elec }} * \mathrm{k}(5)
$$

Where $k$ is the number of bit per packet, $d$ is the distance from the sender node to the receiver node, $E_{\text {elec }}$ and $E_{a m p}$ are per bit energy dissipation in transmitting or receiving circuitry and energy required per bit per meter square for the amplifier to achieve acceptable signal to noise ratio (SNR) respectively. Simulations are done using the values $50 \mathrm{~nJ} / \mathrm{bit}$ and $100 \mathrm{pJ} / \mathrm{bit} / \mathrm{m}^{2}$ for $E_{\text {elec }}$ and $E_{a m p}$, respectively. Table 2 present the systems parameters in details.

TABLE 2. Simulation Parameters

\begin{tabular}{|c|c|}
\hline Parameter & Value \\
\hline Topographical Area (meters) & $100 \mathrm{~m} \times 100 \mathrm{~m}$ \\
\hline Sink location (meters) & $(90,90)$ \\
\hline Number of modes & 100 \\
\hline Limit of transmission distance (meters) & $30 \mathrm{~m}$ \\
\hline Initial energy of node & $0.5 \mathrm{~J}$ \\
\hline$E_{\text {elec }}$ & $50 \mathrm{~nJ} / \mathrm{bit}$ \\
\hline$E_{\text {amp }}$ & $100 \mathrm{pJ} / \mathrm{bit} / \mathrm{m}^{2}$ \\
\hline Packet data size & $2 \mathrm{k} \mathrm{bit}$ \\
\hline Number of transmission packets & $2 \times 10^{4}$ \\
\hline
\end{tabular}

\subsection{Simulation Result}

The number of alive nodes as a function of rounds by using different approaches is shown in Fig. 8.

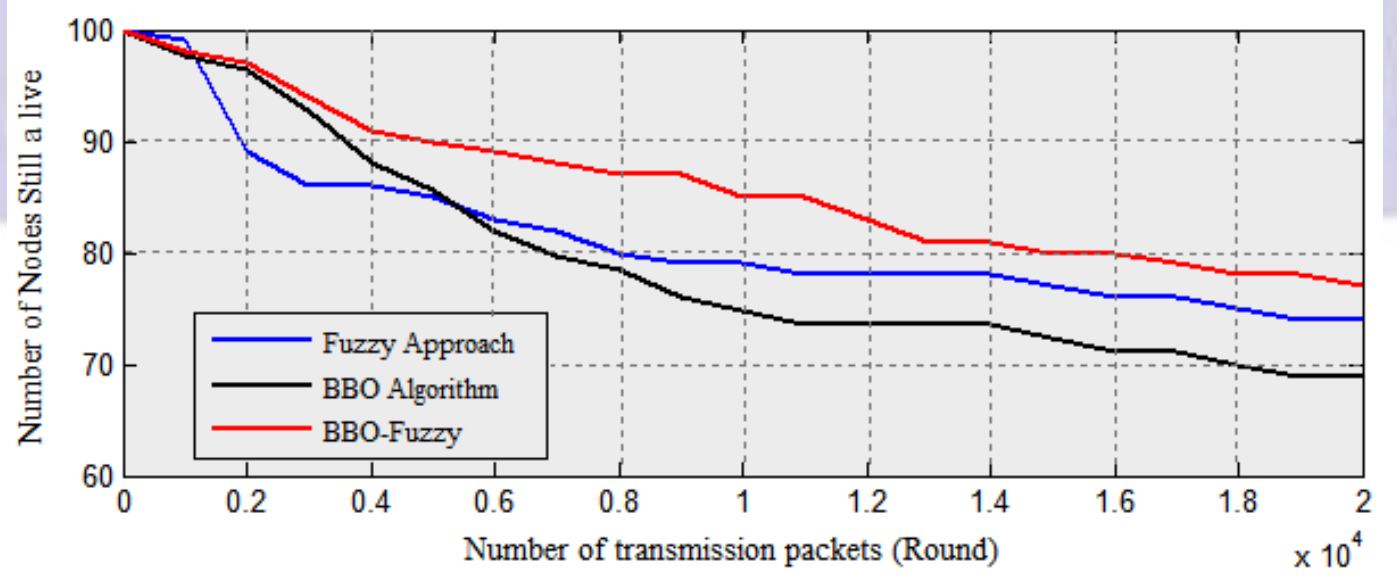

Fig8. Number of alive nodes as a function of rounds

based on different approaches (BBO, Fuzzy and proposed)

The different duration of time corresponding to the first dead node computed using three different approaches is listed in Table 3. From Fig.8 and Table 3, it is clear that, using the proposed method the lifetime of a WSN is longer than the lifetimes of a WSN using BBO algorithm or fuzzy approach. 
Table 3: Number of rounds with the first dead node.

\begin{tabular}{|c|c|c|c|}
\hline Approaches & BBO & Fuzzy & $\begin{array}{l}\text { Proposed } \\
\text { method }\end{array}$ \\
\hline $\begin{array}{c}\text { Lifetime of the first dead } \\
\text { node (Rounds) }\end{array}$ & 1384 & 2373 & 6781 \\
\hline
\end{tabular}

Fig. 9 shows the average remaining energy of a WSN as a function of transmission rounds for the three approaches. As the round number increases, the proposed method performs better than both BBO algorithm and Fuzzy approach. This indicates that, better energy balance in a WSN is achieved by the proposed method.

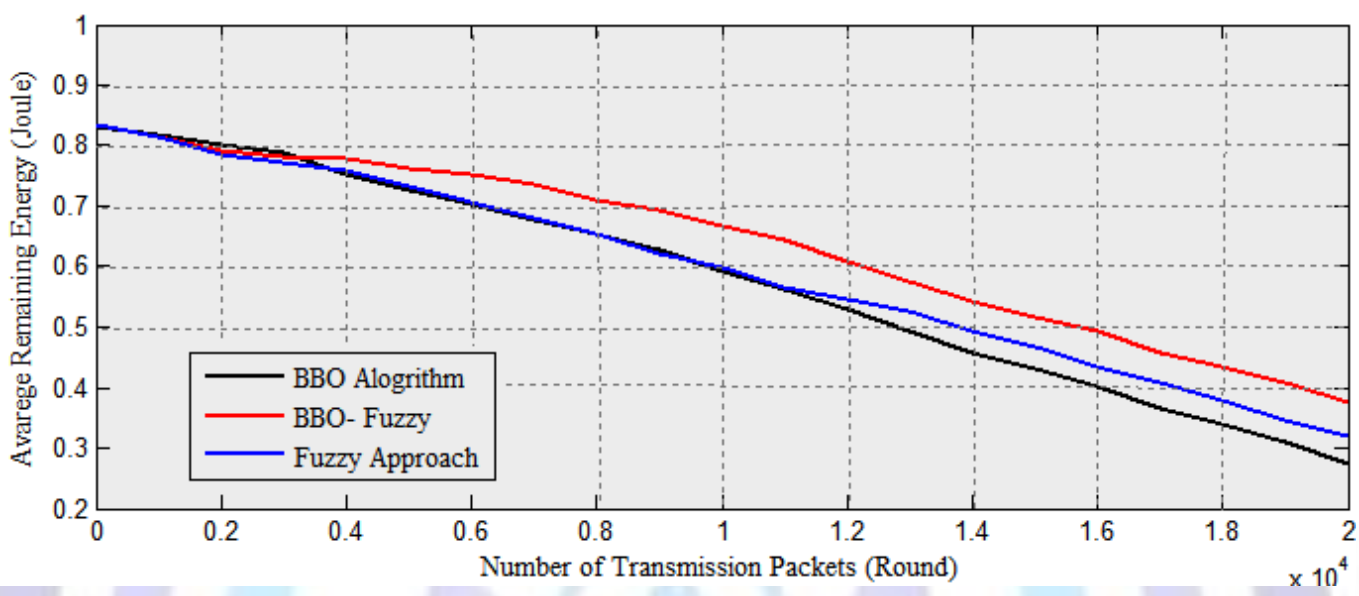

Fig9. Average network remaining energy as a function of transmission round.

\section{CONCLUSION}

Resource limitations have to be taken into account when designing a WSN infrastructure. Energy is one of the most critical resources for WSNs. Most of works in the literatures about WSN routing have emphasized energy savings as an important optimization goal. However, where nodes operate in WSNs on limited battery energy efficient utilization of the energy is very important. The lifetime network is highly related to the route selection, this is one of the main characteristics of these networks. Unbalanced energy consumption is an inherent problem in a WSN. To efficiently route data through transmission path from node to node and to prolong the overall lifetime of the network, we proposed a new algorithm by using a combination of both Fuzzy approach and BBO algorithm. The new method is capable of selecting optimal routing path from the source node to the sink by favoring highest remaining energy and minimum number of hops. The performance of this method is evaluated and compared with other two methods under the same criteria. Simulation results demonstrate the effectiveness of the new approach with regards to enhancement of the lifetime of wireless sensor networks with randomly scattered nodes.

\section{ACKNOWLEDGMENT}

This work was supported in part by the National Natural Science Foundation of China under Grants 61471408; by the National High Technology Research and Development Program ("863"Program) of China under Grants 2014 AA01A701.

\section{.REFERENCES}

[1]Z. Haibo, and $\mathrm{H}$. Shen. ,Balancing energy consumption to maximize network lifetime in data-gathering sensor networks, Parallel and Distributed Systems, IEEE Transactions on 20, no. 10 ,2009,pp. 1526-1539.

[2]C. Hua, T. P. Yum. ,Optimal Routing and Data Aggregation for Maximizing Lifetime of Wireless Sensor Networks, IEEE/ACM Trans Networking, vol.16, no.4,Aug. 2008, pp. $892-903$.

[3]R. Fengyuan, J. Zhang, T. He, C. Lin, and S. K. Ren. ,EBRP: energy-balanced routing protocol for data gathering in wireless sensor networks, Parallel and Distributed Systems, IEEE Transactions on 22, no. 12 ,2011, pp. 2108-2125.

[4]K. Akkaya, M. Younis. ,A survey on routing protocols for wireless sensor networks, Ad Hoc Networks, vol. 3, no. 3, May, 2005, pp.325-349.

[5]S. Haining, Q. Liang, and J. Gao. ,Wireless sensor network lifetime analysis using interval type-2 fuzzy logic systems, IEEE Transactions on 16, no. 2,2008 , pp. 416-427. 
[6]P. Sampath, K. Kinoshita, H. Tode, and K. Murakami. , A clustering/multi-hop hybrid routing method for wireless sensor networks with heterogeneous node types, In GLOBECOM Workshops (GC Wkshps), IEEE, 2010, pp. 207-212.

[7]L. Ying, J. Zhang, H. S. H. Chung, W. H. Ip, Y. Li, and Y. H. Shi. ,An ant colony optimization approach for maximizing the lifetime of heterogeneous wireless sensor networks, Systems, Man, and Cybernetics, Part C: Applications and Reviews, IEEE Transactions on 42, no. 3 ,2012, 408-420.

[8]M. A. Azim and A. Jamalipour.,Performance evaluation of optimized forwarding strategy for flat sensor networks, in Proc. IEEE Global Telecommun. Conf., Nov. 2007, pp. 710-714.

[9]S. Y. Chiang and J. L. Wang.,Routing analysis using fuzzy logic systems in wireless sensor networks, Lecture Notes Comput. Sci., vol. 5178, 2008, pp. 966-973.

[10]K. M. Rana and M. A. Zaveri.,ASEER: A routing method to extend life of two-tiered wireless sensor network, Int. J. Adv. Smart Sensor Netw. Syst., vol. 11, no. 2, Oct. 2011, pp. 1-16.

[11]W. Gaige, L. Guo, H. Duan, L. Liu, and H. Q. Wang. ,Dynamic deployment of wireless sensor networks by biogeography based optimization algorithm, Journal of Sensor and Actuator Networks 1, no. 2 ,2012, pp.86-96.

[12]W. Dargie and C. Poellabauer. ,Network layer in Fundamental of Wireless Sensor Networks Theory and Practice, New York: Wiley, 2010, pp. 163-204.

[13]Y. M. Lu and V. W. S. Wong.,An energy-efficient multipath routing protocol for wireless sensor networks, in Proc. IEEE 64th Vehicular Technol. Conf., Sep. 2006, pp. 1-5.

[14]Z. LotfiA. ,Soft computing and fuzzy logic, IEEE software $11,1994$.

[15]K. Raghavendra V., A. Förster, and G. Kumar V. ,Computational intelligence in wireless sensor networks: A survey, Communications Surveys \& Tutorials, IEEE 13, no. 1,2011 , pp.68-96.

[16] K.Y. Cai and L. Zhang. ,Fuzzy reasoning as a control problem, IEEE Trans. Fuzzy Syst., vol. 16, no. 3, Jun. 2008, pp. 600-614.

[17]A. Imad S., L. Yan, W. Pan, and B. Luo. ,Lifetime enhancement in wireless sensor networks using fuzzy approach and A-star algorithm, Sensors Journal, IEEE 12, no. 10 ,2012, pp. 3010-3018.

[18] S. Dan. ,Biogeography-based optimization, Evolutionary Computation, IEEE Transactions on 12, no. 6 ,2008,pp. 702 713.

[19]H. R. Karkvandi, E. Pecht, and O. Yadid.,Effective lifetime-aware routing in wireless sensor networks, IEEE Sensors J., vol. 11, no. 12, Dec.2011,pp. 3359-3367.

[20]R. Thomas. ,Selection of appropriate defuzzification methods using application specific properties, Fuzzy Systems, IEEE Transactions on 5, no. 1, 1997,pp. 72-79.

[21]W. R. Heinzelman, A. Chandrakasan, and H. Balakrishnan.,Energyefficient communication protocol for wireless microsensor networks, in Proc. 33rd Ann. Hawaii Int. Conf. Syst. Sci., 2000, pp. 1-10.

\section{Author' biography with Photo}

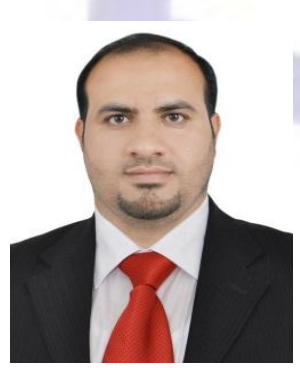

BasimAbood was born in Thi-Qar City, Iraq, in 1984. He received the B.Sc. degree from Al-Basra University, Basra (BU), in electrical engineering, in 2007. He received his M.Sc. degree from Huazhong University of Science and Technology (HUST), China, in 2013, in Telecommunication and Information Engineering. He is currently pursuing the Ph.D. degree with the Department of Electronic and Information Engineering, HUST. His research interests include Wirless sensor Networks, Artificial Intelligence, and Heterogeneous Network.

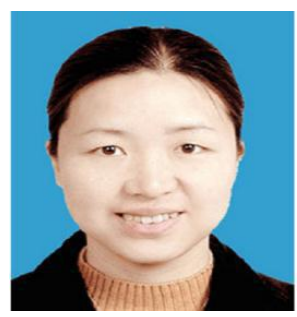

Li Yu was received her B.Sc, M.Sc, Ph.D. degree in Electronic and Information Engineering from Huazhong University of Science and Technology in 1992, 1995, and 1999 respectively. She is now the Dean of Division of Communication and Intelligent Network, Wuhan National Laboratory of Optoelectronic. She is also a chief member of AVS group. Prof. Yu was awarded the University Key. 


\section{ISSN 2277-3061}
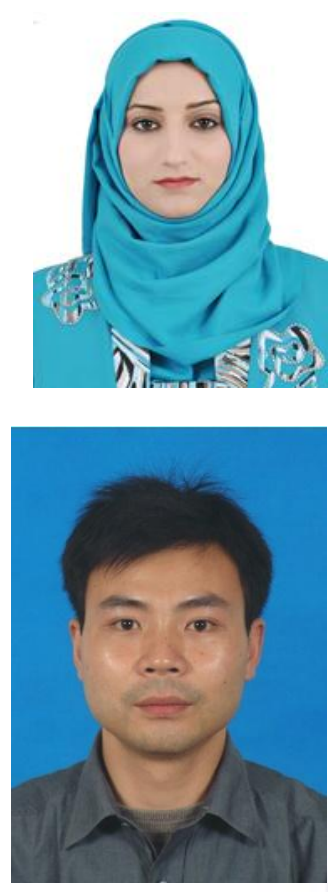

Desheng Wang received his Bachelor degree from Wuhan University of Technology in 1998 M.S. degree from Chinese Academy of Sciences in 2001, and Ph.D. degree from Huazhong University of Science and Technology, majored in Electronics and Information Engineering in 2004. His current research interests lie in the field of sensor network, cooperative MIMO communication, cross layer-based radio link protocol design and so on.

AliaaHussienwas born in Baghdad City, Iraq, in 1987. She received the B.Sc. degree from Baghdad University, Baghdad, in College of Science, Department of astronomy, in 2010. She is currently pursuing the M.Sc. degree Department of astronomy. Her research interests Remote sensing, image processing.

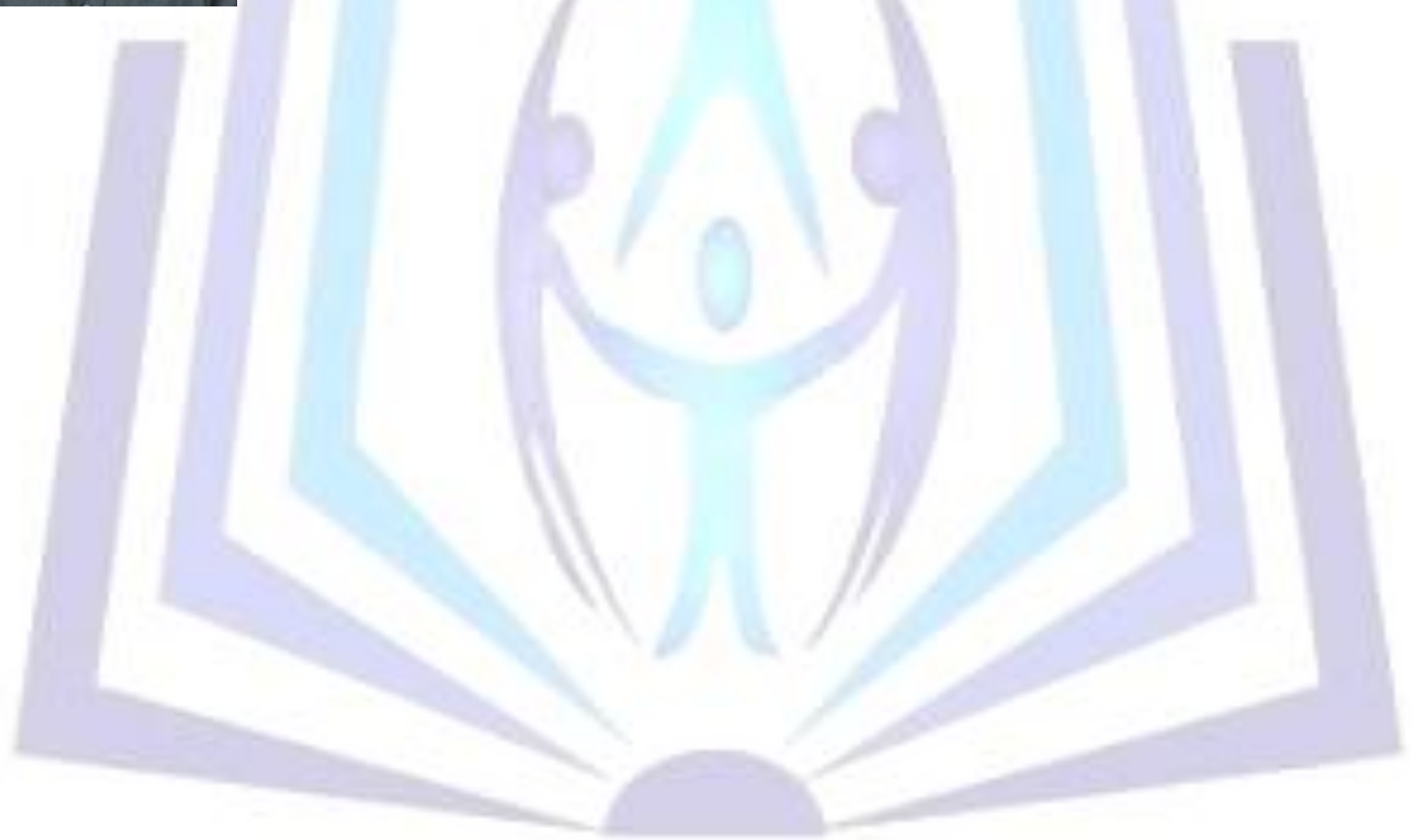

